\title{
La resiliencia como factor fundamental en tiempos de Covid-19
}

\section{Grief and resilience as a fundamental factor in times of Covid-19.}

\author{
Carlos Germán Castagnola Sánchez \\ Universidad César Vallejo, Lima, Perú \\ ORCID: https://orcid.org/0000-0002-2238-328X \\ Juan Carlos-Cotrina \\ Universidad Cesar Vallejo, Lima, Perú. \\ ORCID: https://orcid.org/0000-0003-0293-0394 \\ Dante Aguinaga-Villegas \\ Universidad Nacional Mayor de San Marcos, Lima, Perú \\ Orcid: https://orcid.org/0000-0001-5166-0509
}

Received 10-12-20 Revised 11-25-20

*Correspondence

Email: ccastagnola67@hotmail.com
Accepted 02-13-21 On line 02-27-21

Cite as:

Castagnola, C., Carlos-Cotrina, J., \& Aguinaga-Villegas, D. (2021). La resiliencia como factor fundamental en tiempos de Covid-19. Propósitos y Representaciones, 9 (1), e1044. Doi: http://dx.doi.org/10.20511/pyr2021.v9n1.1044 


\section{Resumen}

En la actualidad, en donde se vive una crisis mundial de salud a causa de un virus Ilamado Covid-19, presentando un gran reto para el campo de la medicina y un cambio drástico en las vidas de las personas, se debe idear un modo diferente de poder llevar el duelo porque el virus sigue vigente en el entorno, además de haber perdido muchas vidas, no se permite realizar un duelo con el respeto y costumbre que se tiene en distintas culturas. A lo largo de la pandemia, se muestran momentos de angustia, dolor, depresión y caos; debiendo superar de manera drástica y resiliente. La presente investigación es de tipo mixta, exploratoria- descriptiva, cuyos resultados se realizan para el contexto académico mediante un muestreo aleatorio simple, en base al cálculo de la muestra y la predisposición de los encuestados de participar en forma voluntaria, teniendo como muestra a 261 personas entre jóvenes y adultos de 20 a más de 60 años. En conclusión, la resiliencia post pandemia se puede advertir en los encuestados que se encuentran proclives a un estado de depresión y ansiedad, el mismo que debe ser atendido por los especialistas brindando un apoyo psicosocial.

Palabras clave: Resiliencia, fortaleza, vulnerabilidad, Covid-19, duelo.

\section{Summary}

At present, where there is a global health crisis due to a virus called Covid-19, presenting a great challenge for the field of medicine and a drastic change in people's lives, a different way must be devised to be able to mourn because the virus is still in force in the environment, in addition to having lost many lives, a duel is not allowed with the respect and custom that is had in different cultures. Throughout the pandemic, there are moments of anguish, pain, depression and chaos; having to overcome drastically and resiliently. The present investigation is of a mixed, exploratory-descriptive type, the results of which are carried out for the academic context by means of a simple random sampling, based on the calculation of the sample and the predisposition of the respondents to participate 
voluntarily, taking as a sample 261 people between youth and adults aged 20 to over 60 years. In conclusion, post-pandemic resilience can be seen in respondents who are prone to a state of depression and anxiety, the same that must be attended by specialists providing psychosocial support.

Keywords: Resilience, strength, vulnerability, Covid-19, grief.

\section{Introducción}

La pandemia ocasionada por el virus del Covid-19, ocasiono medidas de prevención como lavarse las manos, mantener la distancia social y llevar mascarilla, muchas personas han puesto sus esperanzas en una vacuna que permita contrarrestar el virus, sin embargo, aunque se están desarrollando muchas vacunas, aún puede transcurrir mucho tiempo antes de que puedan administrarse a los ciudadanos, mientras tanto las personas se deben enfocar más en la prevención y en la generación de resiliencia (Oltra y Boso, 2020).

En este contexto, el personal sanitario y la población en general, están viviendo el estrés crónico con probables episodios sobrevenidos de estrés agudo, lo que la convierte en potencialmente muy dañina para el equilibrio emocional de nuestro personal sanitario (Barbado Fernández, 2020).

Es por ello, que con el presente artículo se busca describir los distintos problemas y posibles consecuencias que ha ocasionado la pandemia por el Covid-19 en la salud mental de la población, a causa del aislamiento social y la gran cantidad de pérdidas humanas sufridas, además de observar el nivel de respuesta resiliente que ha tomado la población ante la problemática y destacar la importancia de la resiliencia.

La resiliencia es la capacidad de resistir la adversidad y recuperarse de sucesos vitales difíciles. De manera comunitaria es la capacidad sostenida, por parte de un grupo de personas que utilizan los recursos disponibles, como la planificación urbana, el transporte, la alimentación, etc. para responder a los acontecimientos adversos, soportarlos y recuperarse de sus efectos (Bahena-Ayala, Arreguín-Cortés y CervantesJaimes, 2020). Además, es una cualidad universal que ha existido desde siempre, los historiadores la han reflejado al describir como las personas y los pueblos afrontan las adversidades y progresan culturalmente. La palabra resiliencia se refiere a la capacidad de sobreponerse a momentos críticos y adaptarse luego de experimentar alguna situación inusual e inesperada, también indica volver a la normalidad. Resiliencia es un término que deriva del verbo en latín resilio, resilire, que significa "saltar hacia atrás, 
rebotar". Sin embargo, la experiencia nos demuestra lo contrario, es saltar y superar los obstáculos, tal como ha venido sucediendo en la pandemia generada por el Covid-19 (Denckla, C., et. al., 2020; Salanova, 2020).

En el siglo XVIII, el pedagogo suizo H. Pestalozzi observó que niños de la calle, huérfanos y abandonados, mostraban un gran impulso por vivir y salir adelante. (citado en Uriarte, 2005). En Noruega, por ejemplo, a estos niños les llaman "niños diente de león", por su similitud con la planta frágil que crece por todas partes, incluso en medios muy difíciles. En la India les representan con la flor de loto, porque es capaz de nacer en aguas sucias. (Vanistendael y Lecomte, 2002).

Muchos profesionales de la salud y de la educación se han sorprendido al comprobar cómo sujetos que viven situaciones extremadamente difíciles son capaces de sobreponerse y llevar una vida normal, personas que con su actitud y mentalidad han sido capaces de superar positivamente los límites que la ciencia médica les pronosticaba. Esta característica de las personas y de los pueblos ha estado presente seguramente desde los orígenes de la especie humana, pero no ha sido hasta los años ochenta cuando se le ha analizado científicamente (Ochoa, 2020).

El concepto de resiliencia se introduce en la psicología en la década de los ochenta a partir de los estudios de Werner y Smith (1982), en una época en la que predominaba el concepto de vulnerabilidad. Werner evaluó en 1955 a 698 recién nacidos en la isla Kauai en el archipiélago de Hawai. Posteriormente continuó con el seguimiento de 201 niños, que procedían de ambientes socio familiares desfavorecidos y para los cuales se estimaba un futuro desarrollo psicosocial negativo.

Casi treinta años después, E. Werner descubrió que 72 de estos 201 sujetos de riesgo llevaban una vida adaptada y normal, a pesar de no haber contado con ningún tipo de atención especial. Werner y Smith los denominaron resistentes al destino y a la característica común a todos ellos "resiliencia". Posteriormente, Wolin y Wolin (1993) han utilizado el concepto de resiliencia para describir a las personas, niños y adultos, que poseen esa fuerza interior, que incluye las siguientes características: introspección, independencia, capacidad de relacionarse, iniciativa, sentido del humor, creatividad y moralidad (Castilla, et. al.,2017).

Se puede aceptar que la resiliencia forma parte del proceso evolutivo de los individuos, pero no está claro que sea una cualidad innata ni tampoco estrictamente adquirida (Grotberg, 1995). Se entiende mejor como una capacidad que se construye en el proceso de interacción sujeto-contexto (Rutter, 1993) que incluye tanto las relaciones sociales como los procesos intrapsíquicos (motivos, representaciones, 
ajuste), y que en conjunto permiten tener una vida "normal" en un contexto patógeno. Según B. Cyrulnik (2004) todos estamos modelados por la mirada de los demás y depende de esas miradas que uno se construya como resiliente o derive en un inadaptado psicosocial.

La familia resiliente es la que se desenvuelve en una dinámica de interacción positiva, basada en el afecto y el apoyo mutuos, protege a sus miembros de circunstancias negativas del entorno social y estimula la formación y la autonomía de los hijos. De esta manera, ayudándoles a ser competentes como futuros adultos, las familias contribuyen al desarrollo de la resiliencia (Bird, 2005).

Los factores internos del individuo y los factores del entorno inmediato que favorecen la resiliencia son difíciles de diferenciar, pues están íntimamente relacionados. Se sabe que se reducen las fuentes de estrés infantil al poseer una buena constitución física y un sistema inmunitario adecuado, en la ausencia de contagios y enfermedades importantes, cuando las intervenciones médicas son puntuales y de carácter preventivo. Una persona fundamentalmente sana y de apariencia agradable tiene más posibilidades de evolucionar sin trastornos psicológicos que una persona con anomalías físicas, discapacidades y necesitado de cuidados socio-sanitarios (Werner y Smith, 1992). Tanto el desarrollo prenatal como las maneras de realizar la crianza en las primeras edades remiten a las características de los padres, su formación y equilibrio mental. Incluso las actitudes positivas de los padres pueden contribuir a hacer más llevaderas las dificultades derivadas de ciertas limitaciones físicas, discapacidades 0 enfermedades de los hijos.

El temperamento ha sido destacado como uno de los factores constitucionales no inmediatos que favorecen la resiliencia (Werner y Smith, 1992). Los niños de temperamento fácil, los que sonríen, los que agradan a primera vista, los que olvidan con prontitud las pequeñas frustraciones de cada día, son fáciles de educar y atender, y vincularse socialmente de manera adecuada construyen un recurso protector que les puede llevar a ser resilientes. En cambio, el temperamento difícil, asociado a la actividad excesiva o muy escasa, el negativismo, altos niveles de reactividad e irritabilidad supone un riesgo de alteraciones psicológicas posteriores (Del Barrio, 2005). Habría que pensar si lo que llamamos temperamento no está a su vez influido por las condiciones de desarrollo prenatal, las cuales remiten a la salud física y emocional de la madre y a sus condiciones de vida. La relación que confiere al niño sentimientos de seguridad afectiva, altos niveles de autoestima y autoeficacia, promueve la autonomía, establece metas adecuadas a sus características y posibilidades, facilita las relaciones extra familiares y 
la formación de amistades, que es proporcionada por padres de todo tipo de contextos sociales, posibilita superar las adversidades y evitar los trastornos psicológicos. (Uriarte, 2005).

Todas las personas tienen algo de resiliencia y todas pueden promover la resiliencia en cuanto que son responsables de otros y pertenecen al entorno de los demás. Todo lo que contribuye a mejorar las relaciones de calidad con los demás, a comprenderles y aceptarles a pesar de las diferencias, favorece la resiliencia propia y la de los demás (Ezpeleta, 2005).

Cuando el trauma es demasiado intenso, el sistema neurológico adaptativo puede desbordarse. También puede ocurrir que los factores desencadenantes no sean muy graves y a pesar de ello igualmente traumáticos si el sujeto es frágil o se encuentra en una situación de vulnerabilidad al haber acumulado muchas situaciones difíciles. Desde este punto de vista, lo "traumático" se refiere a esta información de la experiencia no asimilada (imágenes, sensaciones corporales, emociones), de carácter disfuncional, que permanece en el cerebro emocional, en un sistema de neuronas propio, desconectado del cerebro racional. Los tratamientos eficaces que ayuden a superar los traumas y a ser más resilientes serán los que faciliten los procesos de autocuración del propio cerebro humano (Servan-Schreiber, 2003; Tomkiewicz, 2004).

La resiliencia se ha destacado como un enfoque positivo de la intervención social con quienes sufren y viven en situaciones de pobreza y exclusión social al movilizar las fortalezas y oportunidades que facilitan el progreso hacia una vida más digna y saludable. La resiliencia adoptada por el hombre durante el desarrollo de la pandemia jugará un rol importante en su desarrollo para el futuro. Tenemos claro que el periodo de encierro, para la mayoría de las personas, inició bien, como un descanso, unas pequeñas vacaciones, pero con el paso del tiempo y las ampliaciones de los plazos la resiliencia de cada persona se vio afectada con diversos problemas, entre ellos el estrés y la ansiedad. Manejar correctamente nuestras emociones, aplicando la resiliencia en nuestras vidas ahora marcará el rumbo de nuestro bienestar personal a futuro.

En estos tiempos de confinamiento obligatorio y en tiempos del COVID-19, la humanidad se ha visto vulnerable al estrés agudo, situación traumática con síntomas semejantes al estrés postraumático; estrés crónico que produce desregulaciones psicobiológicas conduciendo múltiples problemas físicos y mentales; estrés postraumático, trastorno mental que aparece después de una situación traumática. Por otro lado, la ansiedad y el miedo son los combustibles que conlleva la mayor parte de la 
psicopatología. Aquí es importante estar pendientes de su aparición para detectarla a tiempo y solicitar ayuda y poder actuar frente a ella (Lee, Yu y Kim, 2019).

Para ser resilientes se debe tomar algunas consideraciones según Barbado y Fernández, (2020) .a) Aceptación. No es fácil aceptar la situación que la humanidad está viviendo, especialmente aquellos que están en primera línea; pero se tiene que aceptar. Cuanto antes se acepte, menos se resentirá psicológicamente. b) Apoyo mutuo. El trabajo como grupo es fundamental en esta crisis. Buscar momentos para hablar, para escucharse unos a otros, para llorar, para gritar, incluso, para reír. Este sistema de colaboración es importante en estos tiempos que hace sentir la soledad. c) El autocuidado. El gran riesgo psicológico que se corre en esta situación laboral es el agotamiento psicofísico (Tomyn y Weinberg, 2018). Por eso es importante la regulación de energía, con el cuidado así mismo con el cuidado del cuerpo, permaneciendo en lo que se está haciendo, gestionando adecuadamente el tiempo, descargar el estrés con actividades lúdicas o físicas.

Para Lasota, Tomaszek y Bosacki (2020), en su investigación sobre la empatía, resiliencia y gratitud, se probó que el papel de mediación de la resiliencia en las asociaciones entre empatía y gratitud. Según el médico Psicoterapeuta Brik (2020), la resiliencia se puede potenciar a nivel individual en el Covid-19: Manteniéndose activos, reflexivos y controlando las situaciones frente al entorno, potenciando y utilizando las capacidades intelectuales frente a la adversidad, impulsando la cohesión familiar: manteniendo roles, jerarquías, límites y diferenciación entre los subsistemas, demostrando afecto, ternura y preocupación por el bienestar de los niños y de cada uno de los miembros de la familia, disponiendo de apoyo externo: profesores, familiares o amigos significativos, y de instituciones o grupos: escuelas, servicios de salud, servicios sociales, congregaciones religiosas u otros.

\section{Método}

La investigación que se realizará será de tipo mixta, exploratoriadescriptiva, cuyos resultados se realizan para el contexto académico mediante un muestreo aleatorio simple, pues la población encuestada no ha sido seleccionada, si no ha sido escogida de manera aleatoria, en base al cálculo de la muestra y la predisposición de los encuestados de participar en forma voluntaria, siendo el universo tomado entre jóvenes y adultos de 20 a más de 60 años. 
Es evidente que en este tiempo de crisis generado por la pandemia del Covid-19, se ha experimentado sin distinción de edad, lugar, estado civil situaciones negativas de depresión y ansiedad y los signos latentes de ansiedad, es por ello que se escogió el test que permita visualizar el grado de ansiedad y depresión para nuestra muestra, en este estadio debemos pensar y reflexionar como actuar con resiliencia frente a la pandemia Post Covid-19.

La muestra estará constituida por universo de 261 personas encuestadas entre estudiantes universitarios, técnicos y adultos, con un rango de edad entre los 20 y 60 años a más, dividida por nivel económico, lugar de residencia, estado civil, situación económica. El muestreo fue intencional y se realizó a través de una encuesta en de forma online a fin de facilitar el acceso a la población.

El instrumento a utilizar es el de Resilience Scale (RS-14) de 14 items de Walgnild, (2009c), basada en la Resilience Scale (RS-25) de 25 item (Wagnild \&Young, 1993 citado en Laurente, 2019). Este mide el grado de resiliencia individual, considerada como característica de personalidad positiva que permite la adaptación del individuo a situaciones adversas, esta escala correlacionada negativamente con la depresión y la ansiedad (Salgado, 2012).

Es un cuestionario aplicado a 25 items sin embargo, nosotros hemos utilizado los 14 items que evalúa a los encuestados como se han sentido en el último mes durante la pandemia, en una escala de tipo Likert, desde = "nade acuerdo" hasta 4" totalmente de acuerdo" y que nosotros debido al tipo de encuesta online aplicada hemos adaptado del 1 al 5.

El rango de puntuación podrá ser de un máximo de 98 puntos para el factor I: competencia personal y de 30 puntos para el factor II: aceptación de uno mismo y de la vida. Dentro del presente estudio se determinó la fiabilidad de la escala con la obtención del alfa de Cronbach $(0,73)$, la misma que deberá ser analizada. Dentro del presente estudio, se determinó la fiabilidad de la escala con la obtención del alfa de Cronbach. 
Tabla 1

Análisis Factorial Exploratorio de la ER-14

\begin{tabular}{lll}
\hline Factor & $\begin{array}{l}\text { Alfa } \\
\text { Cronbach }\end{array}$ & de \\
\hline F1 & 0,73 \\
\hline F2 & 0,73 \\
\hline
\end{tabular}

RS-14 $\quad 0,73$

Nota: Datos obtenidos de encuestas aplicadas

\section{Resultados}

Posterior al análisis de los datos se obtuvieron que de la muestra se determina que se encuesto a 151 mujeres y 110 hombres. De los dos factores determinados por Walnild y Young son competencia personal y aceptación de uno mismo y la vida. Los cuales son divididos por dos factores.

\section{Tabla 2}

Componentes ER-14

\begin{tabular}{|c|c|c|c|}
\hline & \multirow[b]{2}{*}{ ITEMS } & \multicolumn{2}{|c|}{ DIMENSIONES } \\
\hline \multirow{12}{*}{$\begin{array}{l}\text { COMPETENCIA } \\
\text { PERSONAL }\end{array}$} & & FACTOR I & FACTOR II \\
\hline & 1. Normalmente, me las arreglode una manera u otra & 0,92 & 0,16 \\
\hline & 2. Me siento orgulloso de las cosas que he logrado & 0,89 & 0,12 \\
\hline & 5. Siento que puedo mejorar muchas situaciones a la vez & 0,83 & 0,03 \\
\hline & 6. Soy Resuelto y decidido & 0,90 & 0,05 \\
\hline & $\begin{array}{l}\text { 7. No me asusta sufrir dificultades porque ya las he } \\
\text { experimentado }\end{array}$ & 0,92 & $-0,08$ \\
\hline & 9.Pongo Interés en las cosas & 0,90 & $-0,16$ \\
\hline & 10.Puedo encontrar, generalmente, algo sobre lo que reirme & 0,89 & 0,01 \\
\hline & 11.La seguridad en mismo me ayuda en los momentos dificiles & 0,87 & 0,03 \\
\hline & $\begin{array}{l}\text { 12.En una emergencia, soy alguien en quien la gente puede } \\
\text { confiar }\end{array}$ & 0,85 & 0,05 \\
\hline & 13. Mi vida tiene sentido & 0,88 & 0,14 \\
\hline & $\begin{array}{l}\text { 14. Cuando estoy en una situación dificil, por lo general puedo } \\
\text { encontrar una salida }\end{array}$ & 0,81 & $-0,58$ \\
\hline \multirow{3}{*}{$\begin{array}{l}\text { ACEPTACION } \\
\text { DE UNO } \\
\text { MISMO Y DE } \\
\text { LA VIDA }\end{array}$} & 3.En General me tomo las cosas con calma & 0,83 & $-0,99$ \\
\hline & 4. Soy Una persona con una adecuada estima & 0,91 & $-0,24$ \\
\hline & 8. Soy una persona disciplinada & 0,89 & 0,39 \\
\hline
\end{tabular}


Nota: Sánchez Teruel \& Robles Bello (2015)

Tabla 3

Resultados de encuesta

\begin{tabular}{|c|c|c|c|c|c|c|}
\hline \multicolumn{7}{|c|}{ RESULTADOS ENCUESTA } \\
\hline $\mathrm{F} 1+\mathrm{F} 2$ & MUY ALTA & ALTA & NORMAL & BAJA & MUY BAJA & TOTAL \\
\hline \multirow[t]{2}{*}{$\mathrm{P} 1$} & 172 & 89 & & & & 261 \\
\hline & 1 & 2 & 3 & 4 & 5 & \\
\hline \multirow[t]{2}{*}{$\mathrm{P} 2$} & 112 & 44 & 81 & 21 & 3 & 261 \\
\hline & 1 & 2 & 3 & 4 & 5 & \\
\hline \multirow{2}{*}{ P3 } & 50 & 39 & 83 & 50 & 39 & 261 \\
\hline & 1 & 2 & 3 & 4 & 5 & \\
\hline \multirow[t]{2}{*}{$\mathrm{P} 4$} & 13 & 26 & 84 & 78 & 60 & 261 \\
\hline & 1 & 2 & 3 & 4 & 5 & \\
\hline \multirow[t]{2}{*}{ P5 } & 13 & 18 & 84 & 84 & 62 & 261 \\
\hline & 1 & 2 & 3 & 4 & 5 & \\
\hline \multirow[t]{2}{*}{ P6 } & 11 & 37 & 73 & 78 & 62 & 261 \\
\hline & 1 & 2 & 3 & 4 & 5 & \\
\hline \multirow[t]{2}{*}{ P7 } & 3 & 24 & 75 & 86 & 73 & 261 \\
\hline & 1 & 2 & 3 & 4 & 5 & \\
\hline \multirow[t]{2}{*}{ P8 } & 39 & 34 & 94 & 52 & 42 & 261 \\
\hline & 1 & 2 & 3 & 4 & 5 & \\
\hline \multirow[t]{2}{*}{ P9 } & 6 & 26 & 65 & 78 & 86 & 261 \\
\hline & 1 & 2 & 3 & 4 & 5 & \\
\hline \multirow[t]{2}{*}{ P10 } & 21 & 16 & 68 & 81 & 75 & 261 \\
\hline & 1 & 2 & 3 & 4 & 5 & \\
\hline \multirow[t]{2}{*}{$\mathrm{P} 11$} & 3 & 18 & 60 & 94 & 86 & 261 \\
\hline & 1 & 2 & 3 & 4 & 5 & \\
\hline \multirow[t]{2}{*}{$\mathrm{P} 12$} & 3 & 16 & 39 & 86 & 117 & 261 \\
\hline & 1 & 2 & 3 & 4 & 5 & \\
\hline \multirow[t]{2}{*}{ P13 } & 0 & 6 & 39 & 73 & 143 & 261 \\
\hline & 1 & 2 & 3 & 4 & 5 & \\
\hline \multirow[t]{2}{*}{ P14 } & 13 & 3 & 21 & 63 & 161 & 261 \\
\hline & 1 & 2 & 3 & 4 & 5 & \\
\hline P15 & 3 & 3 & 39 & 75 & 141 & 261 \\
\hline
\end{tabular}

Nota: Datos obtenidos por encuesta 
Tabla 4

Clasificación de respuestas Factor 1: Competencia personal

\begin{tabular}{|c|c|c|c|c|c|c|c|c|c|c|c|c|c|}
\hline \multirow[b]{2}{*}{ Equivalencia P1 } & \multicolumn{13}{|c|}{ FACTOR1: DIMENSION COMPETENCIA PERSONAL } \\
\hline & & $P 2$ & P5 & P6 & P7 & pg & P10 & P11 & P12 & P13 & P14 & $(t) F 1$ & $(-) F 1$ \\
\hline MUY ALTA & $65.90 \%$ & $42.91 \%$ & $4.98 \%$ & $4.21 \%$ & $1.15 \%$ & $2.30 \%$ & $8.05 \%$ & $1.15 \%$ & $1.15 \%$ & $0.00 \%$ & $4.98 \%$ & $65.90 \%$ & $1.15 \%$ \\
\hline ALTA & $34.10 \%$ & $16.86 \%$ & $6.90 \%$ & $14.18 \%$ & $9.20 \%$ & $9.96 \%$ & $6.13 \%$ & $6.90 \%$ & $6.13 \%$ & $2.30 \%$ & $1.15 \%$ & $34.10 \%$ & $1.15 \%$ \\
\hline NORMAL & $0.00 \%$ & $31.03 \%$ & $32.18 \%$ & $27.97 \%$ & $28.74 \%$ & $24.90 \%$ & $26.05 \%$ & $22.99 \%$ & $14.94 \%$ & $14.94 \%$ & $8.05 \%$ & $32.18 \%$ & $8.05 \%$ \\
\hline BAJA & $0.00 \%$ & $8.05 \%$ & $32.18 \%$ & $29.89 \%$ & $32.95 \%$ & $29.89 \%$ & $31.03 \%$ & $36.02 \%$ & $32.95 \%$ & $27.97 \%$ & $24.14 \%$ & $36.02 \%$ & $8.05 \%$ \\
\hline MUY BAJA & $0.00 \%$ & $1.15 \%$ & $23.75 \%$ & $23.75 \%$ & $27.97 \%$ & $32.95 \%$ & $28.74 \%$ & $32.95 \%$ & $44.83 \%$ & $54.79 \%$ & $61.69 \%$ & $61.69 \%$ & 1.1.15\% \\
\hline
\end{tabular}

Nota: Datos obtenidos de encuesta

Del análisis podemos observar que se obtuvo un nivel de resiliencia post covid son normales, es decir, que su nivel de fortaleza para vencer los obstáculos que se esperan a futuro son muy óptimos y favorables encontrándose en una situación de normalidad.

Es así que mostraron un nivel de orgullo dentro de un rango normal por los triunfos obtenidos (p2), así mismo indicaron que sienten que han podido manejar muchas situaciones a la vez durante la pandemia (p3). Asimismo, las personas con un nivel de resiliencia normal y baja, tienen como habilidad importante sacar lo mejor de sí frente a cualquier obstáculo, que para el presente caso fue enfocado a la pandemia.

En cuanto a los rasgos negativos podemos señalar que en un primer momento frente a una situación inesperada de enclaustramiento como fue la pandemia sintieron temor para encontrar una salida (p14). 
Tabla 5

Clasificación de respuestas Factor 2: Aceptación de uno mismo

\begin{tabular}{|c|c|c|c|c|c|}
\hline \multirow[b]{2}{*}{ Equivalencia } & \multicolumn{5}{|c|}{ FACTOR 2: ACEPTACION DE UNO MISMO } \\
\hline & P3 & P4 & P8 & (+) F1 & (-) F1 \\
\hline MUY ALTA & $19.16 \%$ & $4.98 \%$ & $14.94 \%$ & $19.16 \%$ & $4.98 \%$ \\
\hline ALTA & $14.94 \%$ & $9.96 \%$ & $13.03 \%$ & $14.94 \%$ & $9.96 \%$ \\
\hline NORMAL & $31.80 \%$ & $32.18 \%$ & $36.02 \%$ & $36.02 \%$ & $31.80 \%$ \\
\hline BAJA & $19.16 \%$ & $29.89 \%$ & $19.92 \%$ & $29.89 \%$ & $19.16 \%$ \\
\hline MUY BAJA & $14.94 \%$ & $22.99 \%$ & $16.09 \%$ & $22.99 \%$ & $14.94 \%$ \\
\hline
\end{tabular}

Nota: Datos obtenidos de encuesta

En lo que refiere al factor 2: Aceptación de uno mismo, arroja un nivel de resiliencia muy bajo post pandemia tanto en los jóvenes como en los adultos encuestados. Se aprecia que no tienen capacidad de tomar las cosas con calma, no poseen autoestima, no muestran ser disciplinados.

Como consecuencia del análisis de la Resiliencia post pandemia se puede advertir en los encuestados que se encuentran proclives a un estado de depresión y ansiedad, el mismo que debe ser atendido por los especialistas que a continuación se señala brindando un apoyo psicosocial.

\section{Discusión}

Respecto de los resultados obtenidos de la encuesta, y de acuerdo a la metodología a aplicar bajo el contexto de los estudios efectuados a través del ER -14, nos permite efectuar un análisis exhaustivo del grado de depresión y ansiedad generado por la pandemia Covid-19, así como anticipar el grado de resiliencia que tienen los jóvenes y adultos post pandemia. Asimismo, Lasota, Tomaszek y Bosacki (2020), en su investigación sobre la empatía, resiliencia y gratitud, señala que la apertura a nuevas experiencias de vida, la dimensión de resiliencia fue el más predominante en ambos grupos, resultando como parte del análisis un nivel de resiliencia post Covid-19 normal, es decir, que su nivel de fortaleza para vencer los obstáculos que se esperan a futuro son muy óptimos y favorables encontrándose en una situación de normalidad.

En cuanto a los rasgos negativos podemos señalar que en un primer momento frente a una situación inesperada de enclaustramiento como fue la 
pandemia sintieron temor para encontrar una salida. Para Ramírez-Ortiz, CastroQuintero, Lerma-Córdova, Yela-Ceballos y Escobar-Córdoba (2020), en su investigación observo las consecuencias de la pandemia Covid-19 en la salud mental asociadas al aislamiento social, en donde resaltó que trastornos complejos como el insomnio, ansiedad, depresión y estrés postraumático; concluyendo que se requiere que las entidades de salud desarrollen estrategias en aras de soportar al personal de salud y las personas en general, con el propósito de reducir el impacto de las afecciones.

Para Lozano-Diaz, Figueredo, Fernandez-Prados y Martinez (2020), señalaron en su investigación "impactos del confinamiento por el Covid-19" que la resiliencia es importante para sobrellevar el fuerte impacto que ha causado el confinamiento en el aspecto psicológico, laboral y académico.

Así también, Solano (2020) señala que la pandemia del Covid 19 ha ocasionado un complejo problema, que requiere del análisis exhaustivo de diversas disciplinas, como el sector salud, económico, ecológico, bioinformático, entre otros, mediante cambios de paradigmas que fuercen a tener un nuevo estilo de vida y nuevas prioridades.

Además, Espinosa, Mesa, Díaz, Caraballo y Mesa (2020) en su investigación señalan que la pandemia ha tenido un impacto psicológico importante, por lo que la mayoría muestran niveles de estrés altos, y puntuaciones bajas en las dimensiones de resiliencia. Observando que el 79,4 \% mantuvo un comportamiento resiliente mientras que solo un 20,6 \% mostró bajos niveles de resiliencia.

\section{Recomendaciones}

Se recomienda al ministerio de salud el fomento de la resiliencia, la mejora de la salud mental y el pensamiento positivo en el personal de salud y del público en general, dado el contexto crucial que se está viviendo, momentos de dolor por perdidas de familiares cercanos.

Se recomienda al sector educación implementar un currículo socio emocional, en el que los directivos, el maestro, los padres estén involucrado y 
comprometidos con el desarrollo de la resiliencia, la autoestima, la motivación de los estudiantes.

Se recomienda a los centros educativos la implementación de evaluaciones psicológicas y posteriores terapias integrales de ser necesarias, con el fin de soslayar pensamientos automáticos negativos, catastróficos y pesimistas sobre el futuro.

\section{Limitaciones}

Para la investigación se presentó como limitación el proceso de obtención de datos, ya que el aislamiento dificultó la recaudación.

Se observó el aumento de información proveniente de fuentes no confiables, confundiendo a los usuarios y fomentando el temor, asimismo, difundiendo el uso de tratamientos no aprobados científicamente. 


\section{Referencias}

Bahena-Ayala, R., Arreguín-Cortés, F. y Cervantes-Jaimes, C. (2020). Assessing resilience of cities to hydrometeorological hazards. Tecnología y Ciencias del Agua, 12(1). http://revistatyca.org.mx/tca/wpcontent/uploads/2020/proximos_numeros/enero_febrero_2021/2107_fin al.pdf

Barbado, J. A. y Fernández, M. (2020). MIMAPA - Centro de Psiquiatría y Psicología. 1-16. https://www.semg.es/images/2020/Coronavirus/guia_resilencia_estres_ covid19.pdf

Bird, H.R. ( 2005). Pobreza y Nivel socioeconómico. En L. Ezpeleta (Ed): Factores de riesgo en psicopatología del desarrollo, Cap. 9 , pp. 225235. Barcelona: Masson

Brik, E. (2020). Resiliencia en Tiempos del Covid 19 desde el Modelo Sistémico. https://itadsistemica.com/resiliencia/resiliencia-covid19estrategia-afrontamiento/

Castilla, H., Coronel, J., Bonilla, A., Mendoza, M. y Barboza, M. (2017). Validez y Confiabilidad de la Escala de Resiliencia (Scale Resilience) en una muestra de estudiantes y adultos de la Ciudad de Lima. Revista Peruana De Psicología Y Trabajo Social, 4(2), 121-136. https://doi.org/revistas.uigv.edu.pe/index.php/psicología/article/riew/

Denckla, C., Cicchetti, D., Kubzansky, L., Seedat, S., Teicher, M., Williams, D. y Koenen, K. (2020). Psychological resilience: an update on definitions, a critical appraisal, and research recommendations. European Journal of Psychotraumatology, 11 (1), 1822064. https://doi.org/10.1080/20008198.2020.1822064

Del Barrio, V. (2005). Temperamento. En L. Ezpeleta, Ed.: Factores de riesgo en psicopatología del desarrollo. Barcelona: Masson.

Espinosa, Y., Mesa, D., Díaz, Y., Caraballo, L. y Mesa, M. (2020). Estudio del impacto psicológico de la COVID-19 en estudiantes de Ciencias 
Médicas, Los Palacios. Revista Cubana de Salud Pública.46.(Ed especial). https://scielosp.org/pdf/rcsp/2020.v46suppl1/e2659/es

Ezpeleta, L. (ed.) (2005): Factores de riesgo en psicopatología del desarrollo. Barcelona: Masson

Grotberg (1995). A guide to promothing resilience in children. La Haya: Fundación Bernard Van Leer.

Ochoa García, Elena (2020). 10 Características de las personas resilientes. https://coaching-gestalt.com/eres-resiliente-10-caracteristicas-de-laspersonas-resilientes

Oltra, C., y Boso, A. (2020). Lecciones aprendidas de la crisis del coronavirus: preparación y resiliencia social. Revista Española De Sociología, 29(3). https://doi.org/10.22325/fes/res.2020.50

Laurente, G. (2019). Propiedades psicométricas de la escala de resiliencia de Walnild y Young en docentes de Nuevo Chimbote. Escuela Profesional De Psicologia, 12-44.

Lasota, A., Tomaszek, K., y Bosacki, S. (2020). Empatía, resiliencia y gratitud: ¿hay diferencias de género?. Anales De Psicología / Annals of Psychology, 36(3), 521-532. https://doi.org/10.6018/analesps.391541

Lee, D., Yu, E. y Kim, N. (2019). Resilience as a mediator in the relationship between posttraumatic stress and posttraumatic growth among adult accident or crime victims: the moderated mediating effect of childhood trauma. https://doi.org/10.1080/20008198.2019.1704563

Lozano-Díaz, A., Fernández-Prados, J., Figueredo, V. y Martínez, A. (2020). Impactos del confinamiento por el COVID-19 entre universitarios: Satisfacción Vital, Resiliencia y Capital Social Online. International Journal of Sociology of Education, 0, 79-104. doi:http://dx.doi.org/10.17583/rise.2020.5925

Uriarte, J. (2005). La resiliencia. Una nueva perspectiva en psicopatología del desarrollo. Revista de Psicodidáctica, 10 (2), 61-80. https://www.redalyc.org/pdf/175/17510206.pdf 
Ramírez-Ortiz, J., Castro-Quintero, D., Lerma-Córdova, C., Yela -Ceballos, F. y Escobar-Córdoba, F. (2020) Consequences Of The Covid-19 Pandemic In Mental Health Associated With Social Isolation. Scielo. https://preprints.scielo.org/index.php/scielo/preprint/view/303/358

Rutter, M. (1993). Resilience; some conceptual considerations. Journal of Adolescent Health. 14, 8, págs. 626-631

Salanova, M. (2020). How to survive COVID-19? Notes from organisational resilience. International Journal of Social Psychology, 35:3, pages 590599. https://doi.org/10.1080/02134748.2020.1795397

Salgado, C. (2012). Medición de la Resiliencia en Adolescentes y Adultos. Revistas UNIFE, Temática Psicológica. 8(1).

Sánchez, D. y Robles, M. (2015). Propiedades Psicométricas de la versión en español. Revista Iberoamericana De Diagnostico Y Evaluación- $E$ Avaliacao Psicológica Portugal, 2(40), 103-113.

Servan-Schreiber, D. (2003). Curación emocional. Barcelona: Círculo de Lectores

Solano, A. (2020). Resiliencia y Covid 19. Rev. Colomb. Obstet. Ginecol ;71(1). https://revista.fecolsog.org/index.php/rcog/article/view/3531

Tomkiewicz (2004). El buen uso de la resiliencia cuando la resiliencia sustituye a la fatalidad. La resiliencia: resistir y rehacerse. 289-300. https://dialnet.unirioja.es/servlet/articulo?codigo $=1032222$

Tomyn, A., y Weinberg, M. (2018). Resilience and subjective wellbeing: A psychometric evaluation in young Australian adults. Australian Psychologist, 53(1), 68-76. https://doi.org/10.1111/ap.12251

Vanistendael, S. y Lecomte, J. (2002). La felicidad es posible. Despertar en niños maltratados la confianza en sí mismos: construir la resiliencia. Barcelona: Gedisa.

Werner, E.E. y Smith, R.S. (1982). Vulnerable but invicible. A longitudinal study of resilient children and youth. Nueva York: McGrawHill. 
Werner, E.E. y Smith, R.S. (1992). Overcoming the Odds: Hig-Risk Children from Birth to Adulthood. Ithaca. Nueva York: Cornell University Press

Wolin, S.J. y Wolin, S. (1993). The Resilient Self: How Survivors of Troubled Families Rise above Adversity. Nueva York: Villard Books 